\title{
Editorial: Recent Advancements in $X-R a y$ and Neutron Imaging of Dynamic Processes in Earth Sciences
}

\author{
Lucia Mancini ${ }^{1,4}$, Fabio Arzilli ${ }^{2}$, Margherita Polacci ${ }^{2}$ and Marco Voltolini ${ }^{3 *}$ \\ ${ }^{1}$ Elettra-Sincrotrone Trieste S.C.p.A., Trieste, Italy, ${ }^{2}$ School of Earth and Environmental Sciences, University of Manchester, \\ Manchester, United Kingdom, ${ }^{3}$ Lawrence Berkeley National Laboratory, Berkeley, CA, United States, ${ }^{4}$ Lund Institute for Neutron \\ and X-Ray Science, Lund, Sweden
}

Keywords: 4D imaging, X-ray computed tomography, neutron imaging, volcanic systems, fluid transport, porous rocks

Editorial on the Research Topic

Recent Advancements in X-Ray and Neutron Imaging of Dynamic Processes in Earth Sciences

Earth is a dynamic system; therefore, the study of geological processes in function of time is of paramount importance. Also, geological systems often evolve at non-ambient conditions, thus limiting the applications of the most common imaging techniques.

Many processes in different scenarios: volcanic, metamorphic, and reservoirs, but also man-made cement-based materials and ceramics, are inherently dynamic and require an investigation in $4 \mathrm{D}$ (three-dimensions, 3D + time) to be fully understood. These processes are subject to changes due to mechanical stress, reactive transport, heating, etc. The high-penetrating power of hard X-rays and neutrons, coupled with the recent advances in instrumentation and computational methods, fostered the growth of novel imaging approaches, making microradiography and computed microtomography (microCT) unique tools to observe internal structures of materials undergoing different processes.

This Research Topic is focused on hard X-ray and neutron-based imaging studies carried out under controlled conditions, representing the next generation of in-situ time-resolved experiments, with applications in different fields of Earth Sciences, as shown in this Research Topic.

A large number of contributions are related to the investigation of fluid flow and transport properties in porous rocks. The high sensitivity of neutrons to hydrogen-based fluids has been exploited by Cordonnier et al. to investigate the subsurface transport of pollutants, such as cadmium, in limestone samples by using in-situ neutron imaging. Their experiments allow constraining parameters such as local hydraulic conductivity and sorption properties of the dissolved cadmium by the solid matrix, showing that cadmium transport follows preferential pathways. Dynamic neutron imaging has been also used by Zambrano et al. to characterize the geometrical properties of the pore network in carbonate reservoirs. In this study, the complementary results provided by neutron and $\mathrm{X}$-ray imaging are used as an input for computational fluid dynamics simulation. This integrated approach provided novel information about fluid storage and migration in reservoir rocks.

The mineral dissolution in porous diamond has been analyzed by Eckley and Ketcham using laboratory-based X-ray microCT during a sequential acid leaching procedure. The formation of fluorides during acid digestion, which can persist throughout acid leaching procedures, is observed. This can drastically affect yields for geochemical measurements of certain elements. Different segmentation procedures are tested showing that the utilization of CT number information to discern partial porosity below the resolution limit is more reliable than purely binary segmentation.

Arzilli F, Polacci Vancements in X-Ray and Neutron Imaging of Dynamic Processes in

Earth Sciences.

Front. Earth Sci. 8:588463.

doi: 10.3389/feart.2020.588463 
Finally, the comparison of mass and X-ray attenuation loss allowed estimating the relative heavy-metal content of the leached material.

Tonai et al. propose a new systematic method for quality control of X-ray CT data (based on downhole radiodensity profiles from mean CT-values) to analyze core samples from scientific drilling. Thanks to this approach, lithologies and deformation structures can be identified within a geological core, with the potential for an automatic procedure. Numerical measures to CT slices are proposed in order to obtain data reflective of core quality. This method has been applied to data from International Ocean Discovery Program Expedition 370 Site C0023 located at the toe of the Nankai accretionary complex.

Two different works involve studies on Opalinus Clay (OPA). Stavropoulou et al. features a combined X-ray and neutron study about water absorption in a OPA core. The imbibition front advancement is visualized and its effect on the sample microstructure (swelling) is studied via digital volume correlation, providing important information about the use of the material as a sealant. Voltolini and Ajo-Franklin instead addresses the closure of a single fracture in an OPA mini-core with synchrotron $\mathrm{X}$-ray microCT, under flow of $\mathrm{CO}_{2}$-saturated water in a mini-triaxial cell. The study identified the different phenomena involved in the evolution of the sample (swelling, fracturing, generation of choke points, etc.) in function of hydration and stress, and quantified their role on the progressive loss of fracture conductivity.

Marone et al. describe the current hardware and software capabilities in X-ray imaging available at the TOMCAT beamline of SLS (PSI, Switzerland) and highlight their relevance for Earth Sciences studies providing three different examples. 1) $4 \mathrm{D}$ visualization of solute transport in a synthetic porous medium in a flow cell, following the evolution of the solute plume. 2) Deformation of a complex evaporate sample at subsurface $\mathrm{P} / \mathrm{T}$ conditions in a heated triaxial cell. The authors identify the mechanisms underlying the microstructure changes in the sample. 3) Magmatic processes at high temperature, focusing on the deformation of bubbles in foamed volcanic rock samples, in a laser-heated cell with torsional deformation capabilities.

Tripoli et al. and Dobson investigate the effect of strain-rate and associated deformation on magma at high temperature and ambient pressure. The former paper reports how strain-rate increases crystallization kinetics in basaltic magmas compared to a magma that is not deformed. Deformation increases crystal nucleation and growth rates, and decreases incubation time. Increasing strain-rate, however, does not show a discernible effect on crystallization kinetics. The latter presents an experimental apparatus and analytical toolkit for the in situ $\mathrm{X}$-ray tomography based quantification of magmatic microstructural evolution during rheological testing, showing how textures evolve heterogeneously within a shearing magma. Both contributions have implications on magma rheology, and, therefore, on magma transport dynamics and eruption initiation/ cessation.

Finally, in this Research Topic the combination of 2D X-ray chemical mapping with laboratory-based 3D X-ray microCT was applied to the study of metamorphic transformations and fabric evolution in Western Italian Alps (Corti et al.). The resulting parameters allows them to perform dynamic modeling of metamorphic processes showing that samples preserve $100 \%$ of the pre-alpine granulite fabric, while the alpine eclogitic static assemblage corresponds to $78 \%$ though no new fabric is developed.

The examples of 4D imaging presented in this Research Topic clearly display how this approach can reveal unique information about dynamic processes in very diverse branches of Earth Sciences, such as volcanology, structural geology, hydrogeology. Future developments both in the hardware (radiation source, in situ cells, combined techniques) and software (image processing, digital rock physics, modeling) fields will further open new opportunities to understand complex evolving systems.

\section{AUTHOR CONTRIBUTIONS}

All authors listed have made a substantial, direct and intellectual contribution to the work, and approved it for publication.

\section{FUNDING}

MV was supported by DOE-BES under Award \# DE-AC02$05 \mathrm{CH} 11231$.

\section{ACKNOWLEDGMENTS}

We thank the Editorial Board and Editorial Office of Frontiers in Earth Science for the kind invitation to edit this Research Topic and their support in organizing and managing the topic.

Conflict of Interest: The authors declare that the research was conducted in the absence of any commercial or financial relationships that could be construed as a potential conflict of interest.

Copyright (c) 2020 Mancini, Arzilli, Polacci and Voltolini. This is an open-access article distributed under the terms of the Creative Commons Attribution License (CC $B Y$ ). The use, distribution or reproduction in other forums is permitted, provided the original author(s) and the copyright owner(s) are credited and that the original publication in this journal is cited, in accordance with accepted academic practice. No use, distribution or reproduction is permitted which does not comply with these terms. 